\title{
Editorial
}

Theme: Adding the T to ADME: Predictive Toxicity in Renal Drug Development

Guest Editors: Rosalinde Masereeuw and Raymond Lai

\section{Adding the T to ADME: Predictive Toxicity in Renal Drug Development}

\author{
Raymond E. Lai ${ }^{1}$ and Rosalinde Masereeuw ${ }^{2,3}$ iD
}

Received 11 April 2018; accepted 23 July 2018; published online 5 September 2018

Considerable attention has been brought forth toward the ongoing issue of successfully pushing drugs out onto the market with seven out of eight drugs in the pharmaceutical pipeline failing to reach the clinic. Most of the reasons for this are due to a novel compound's propensity to cause more harm than benefit to the patient (e.g., cardiotoxicity, eliciting an immune response, nephrotoxicity, hepatoxicity, etc.). Initial information of a new drug is acquired from studies typically performed in animal models that can differ quite drastically to those of humans in terms of their physiology and drug disposition. This suggests that the accuracy of the pharmacokinetic and pharmacodynamic information obtained for a drug is highly dependent on clinical trial results. The necessity of improved models systems for predicting a drug's bioavailability, safety, and efficacy cannot be overstated and has been widely recognized.

Within AAPS, the Cellular \& Molecular Toxicology Focus group provides a cross-disciplinary forum for all aspects related to drug safety research. The focus group organized a symposium titled "Adding the $\mathrm{T}$ to ADME: predictive toxicity in drug development" held at the 2017 AAPS Annual Meeting held in San Diego, California, USA, on November 15, 2017, which comprised three presentations followed by a panel discussion. The presenters were coauthor Rosalinde Masereeuw, Ph.D.; Michael B. Bolger, Ph.D., Chief scientist at Simulations Plus, Inc.; and Patricia Ryan, Ph.D., Director of Toxicology at MedImmune, LLC. The symposium gathered scientists from across a variety of research settings (e.g., regulatory, academia, industry) in order to shed light on the recent significant progress made in the development of in vitro as well as in vivo models and to

Guest Editors: Rosalinde Masereeuw and Raymond Lai

${ }^{1}$ Department of Pharmaceutics, Virginia Commonwealth University, Richmond, Virginia 23298, USA.

${ }^{2}$ Division of Pharmacology, Utrecht Institute of Pharmaceutical Sciences, Utrecht, The Netherlands.

${ }^{3}$ To whom correspondence should be addressed. (e-mail: R.Masereeuw@uu.nl) provide a discussion on their current and future drug development applications.

This Special Issue of AAPS Journal purposely addresses one of the topics presented at the symposium: the development of novel tools for nephrotoxicity testing. Even with the knowledge on functional proteins expressed by renal tubules and their susceptibility to mediate drugs, currently used in vitro screens are poorly predictive of nephrotoxicity in humans. The lack in translation comes from the inadequate replication of the morphology and function of kidney tubules in cell culture models. Recent advances in protocols that enable the directed differentiation of (stem) cells to specific renal cell types in addition to the development of microfluidics and 3D culture systems have opened a range of potential new platforms for evaluating drug nephrotoxicity. Here, the OrganoPlate as a novel high-throughput 3D microfluidic in vitro model, consisting of 40 fully polarized renal proximal tubules, and its application in drug transport and nephrotoxicity testing, is presented.

Vormann et al. (1) describe the development of this platform with clear tubular epithelial characteristics. Furthermore, a dose-dependent disruption of the epithelial barrier was observed after exposure to the nephrotoxicant cisplatin, which coincided with an increase in effluent LDH activity, and in expression of the tight-junction protein zonaoccludence 1, actin and a DNA-damage marker. Method validation further included drug-drug interactions studies as presented by Vriend and coworkers (2), focusing on two important efflux mechanisms in renal proximal tubule, viz. Pglycoprotein and the combined activities of Multidrug Resistance Proteins 2 and 4. For robust analysis of data, a userfriendly method by means of open-source software compatible with high-throughput screening was developed. Finally, Suter-Dick and colleagues (3) focused on biomarkers of toxicity screening using the platform and presented miRNA's as potential sensitive biomarkers of early damage to tubular epithelial cells caused by several nephrotoxicity mechanisms. Overall, the platform developed might meet the needs of future drug development processes in preclinical phases, 
eventually limiting the numbers of animals used for these purposes.

\section{REFERENCES}

1. Vormann MK, Gijzen L, Hutter S, Boot L, Nicolas A, van den Heuvel A, et al. Nephrotoxicity and kidney transport assessment on 3D perfused proximal tubules. AAPS J. 2018;20(5):90.
2. Vriend J, Nieskens TTG, Vormann MK, van den Berge BT, van den Heuvel A, Russel FGM, et al. Screening of drug-transporter interactions in a 3D microfluidic renal proximal tubule on a chip. AAPS J. 2018;20(5):87.

3. Suter-Dick L, Mauch L, Ramp D, Caj M, Vormann MK, Hutter $\mathrm{S}$, et al. Combining extracellular miRNA determination with microfluidic 3D cell cultures for the assessment of nephrotoxicity: a proof of concept study. AAPS J. 2018;20(5):86. 